\title{
OOST: An Object Oriented Tool for Satellite Orbit Design
}

\author{
${ }^{1}$ Salem A.A., ${ }^{2}$ M. Zaki and ${ }^{3}$ A.A. Wahdan \\ ${ }^{1}$ Ain Shams University, Cairo, Egypt, ${ }^{2}$ A.I., Azhar University, Cairo, Egypt \\ ${ }^{3}$ Systems and control, Ain Shams University, Cairo, Egypt
}

\begin{abstract}
In this study the problems of satellite orbit design and simulation are tackled and an object oriented satellite tool OOST is developed. The tool with its three main modules enables the user to plan and design different satellite missions. It performs all orbital calculations needed, simulates the target orbit and performs analysis on the current active satellite(s). The simulation module of OOST has been used to provide orbit design and satellite simulation for all geocentric orbits using an interactive world map that is synchronized with simulation time. OOST uses a predefined library of orbital mechanics functions to perform the required calculations. Also it allows users to call the library in their own external programs. A satellite database, containing data from more than 900 active satellites, has been linked to OOST in order to allow comparisons of different satellites. A Data Mining technique is applied to the satellite data. The technique as such relies upon the Decision Tree. It leads to discover valuable data related to classifying satellites and defining the trends of their owners. This classification is based on classifying satellite orbits into two trends, one representing the majority of owners and the other represents special cases. These orbits have been analyzed using different simulation capabilities of the Ground Track Module. The applications that have been studied in this study are communication, remote sensing and military missions.
\end{abstract}

Key words: Simulation, visualization, decision tree data mining, orbit design, ground tracks, mission analysis, orbital period

\section{INTRODUCTION}

There are many satellite tools in the field of Orbital Mechanics. Most of these tools are simulators for satellite motions in their orbits ${ }^{[1-4]}$. Some of them include satellite databases with different search capabilities [STK]. Few of them can deal with parabolic and hyperbolic orbits, perform plan change calculations, calculate communication swath width, field of view and some types of perturbations that affect the orbit ${ }^{[5-8]}$. However, the available tools include neither comprehensive orbital mechanics calculations nor data mining capabilities.

OOST has been developed to perform orbit design and simulation for geocentric orbits and solves the problems of orbit errors in two steps as follows:

1. The first step is to establish a library of functions to perform orbital calculations using an object oriented programming language. This library can be used as a separate Run Time Unit, which can be invoked via any software package programmed with object oriented Pascal ${ }^{[9]}$.

2. The second Step is to design and implement a visual computational tool named Object Oriented Satellite Tool OOST that works in the field of Orbital Mechanics. It acts as an Object Oriented, Knowledge Based, tool for Satellite Orbit Design and Simulation.

This tool performs its functions via three modules that allow it to do the following:
* To calculate Orbital Mechanics functions and to represent them in a graphical way that describes clearly the methods and Algorithms of calculations. It can perform orbital calculations such as orbital period, orbit inclination, argument of perigee, true anomaly, satellite velocity, eccentricity, orbital inclination ... etc..

* To simulate the following:

* Ground track of the designed Orbit

* Sensor swath width

* Communication swath width

* Satellite motion around the Earth either by changing its true anomaly or its date and time

* To provide a data mining tool that allows analyzing the design of many satellite orbits from a satellite database, which contains more than 900 currently active satellites using the Decision Tree technique; thus leading to the knowledge of much valuable information about various cases in the field of Orbital Design and generating reports about abnormal orbits.

Orbital Computations: Due to the importance of orbit design process, the proposed software tool are specially designed to act as a simulation tool that avoids any error in orbit design. The orbital computations $^{[6-8]}$, as such, represent the core of the first module of OOST. 


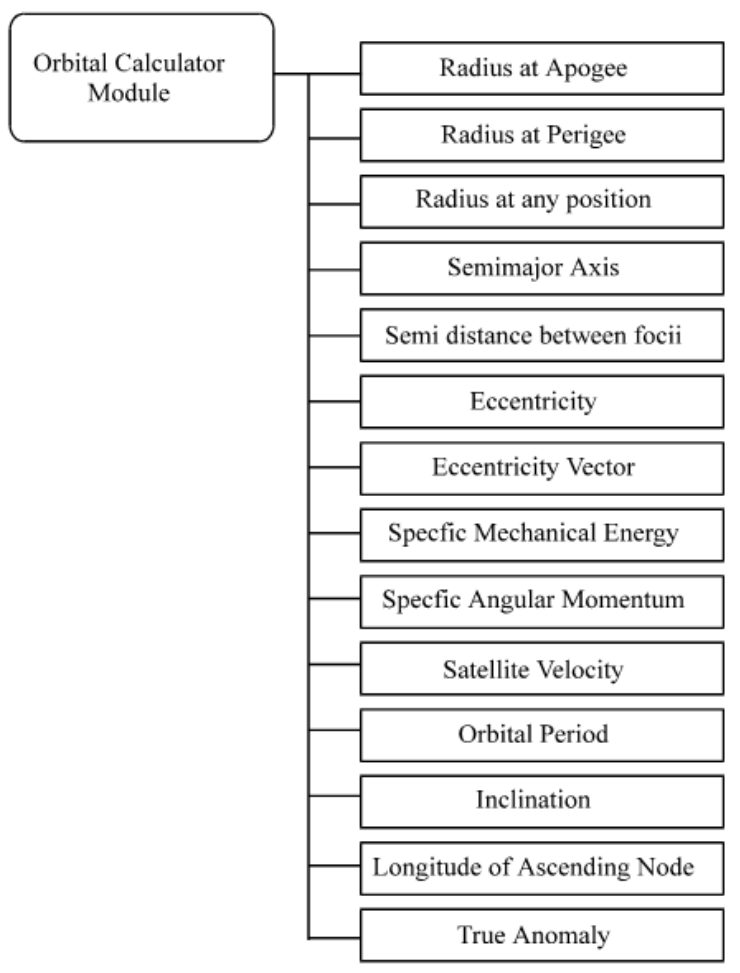

Fig. 1: Items of Orbital Calculation Module (First Module)

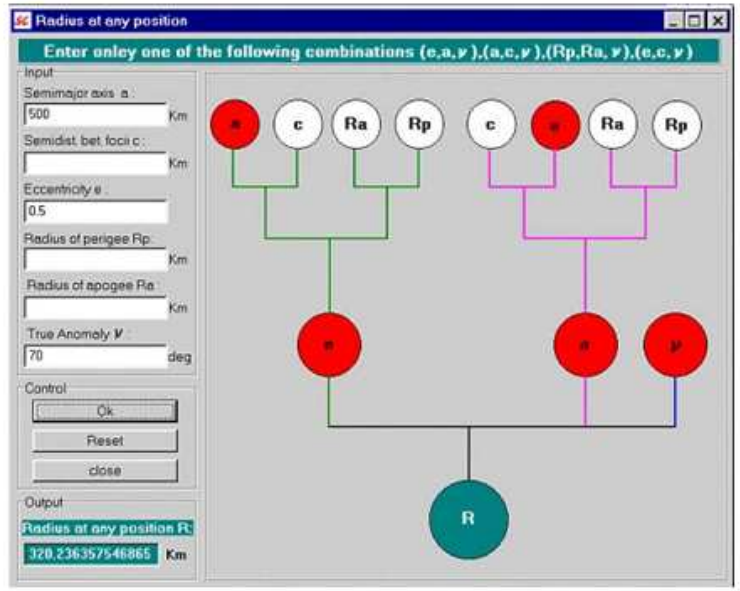

Fig. 2: An Example of One Item that Shows Different Node States

The Library of Orbital Mechanics Functions: A library of orbital mechanics functions "SatOrbits" has been developed to work as a stand-alone library that can be invoked by this package and any other package programmed with object oriented Pascal language. It contains 69 orbital mechanics functions covering all calculations needed for geocentric orbit design and simulation. By using this library, there is no need to include any programming.
Description of the Orbital Calculation Module: Orbital Calculation Module, Fig. 1, simplifies the use of the library of functions (Sat Orbits) and makes it easy to understand the methods of any orbital calculation through its main menu, which contains fourteen forms for calculating fourteen different items. Each item is divided into many algorithms that differ from each other according to the input data available to the user. Any one of these forms contains five groups. The first is used to declare to the user the input combinations that can be entered in order to perform the required calculations; the second is for input data, knowing that entering data in accordance with the declaration mentioned in the first group; the third is for controlling the processing of the program; the fourth is for graphical representation; finally the fifth is for displaying the result of the calculation process.

Orbits Visualization: Different visualization techniques ${ }^{[10,11]}$ are used in this module to allow the user to understand and to monitor changes and improvements of numbers and values representing each category (Orbit shape, orbit orientation, satellite altitude classification, ... etc.). These visualization techniques present tools and aids in exchanging, manipulating and extracting data required in the orbital mechanics field by the satellite mission designer.

An interactive tool has been developed to provide the users with flexibility in choosing their preferable calculating methods, in addition to facilitating calculation steps in a speedy manner through selecting the optimum steps for reaching the target value (Fig. 2).

The tool is named User Guiding Tree (UGT). Each UGT is a tree structure, which consists of main nodes represented by circles and dropped down in hierarchical form to reach the leaf levels that are represented by smaller circles.

The UGTs are well defined to match the mission designer requirements in choosing the calculation method. As soon as a value is entered in any editing area, the UGT starts to detect the validity of the inputs, the readiness of roots or paths to be evaluated or processed and nodes which their values can be automatically inherited from higher level nodes to lower level nodes, in order to generate the target value $^{[10]}$.

The UGT is supplied with a coloring scheme, which is used to inform the user with the entered values, the processing level and to define the nodes required to complete the selected path. Any change in the input values will directly affect the UGT coloring scheme. The user is capable to move to and from any level across nodes and leaves, depending on the availability of parameters.

The UGT is fully recorded with hidden scientific formulas and information required in main and sub levels; unless they are requested by the user, in order to be a guide in all processing stages with suitable 
equations, laws and needed substitutions of variables for reaching a specific target. An abstract is also used on all nodes and leaves, representing the variables required by these nodes.

Object Orientation of UGT: The tree structure of this module consists of circles (Nodes) distributed in the tree leaves. Each circle is an object class named "Node". Each Node has one or two characters inside it. These characters are an abstraction of the underlying variables (for example: "e_Node" means eccentricity object class, " $\Omega$ _Node" means an argument of perigee object class, "a_Node" means semi major axis object class ... etc. "e_Node" is an instance of the object class "Node").

Each "Node" has a state and behavior. The state in this case is either red circles or white circles, which clarify whether the user has entered the object class value or not. The characteristics expressed by the language are as follows:

* A method named "Get Result" is used to calculate the value of instances represented by each object class.

\{ User request: Get Result ,

Check requested object class,

Check available inputs,

Adjust objects colors,

Select the suitable root from the UGT,

Perform required calculations, use inheritance if possible,

Change the output object color to green

Display result to the user

* A method named "Get Info" informs the user with laws, formulas and units used to calculate a certain variable (instance).

$\{$ User request: GetInfo,

Check which instance of the object class is requested,

Display laws, formulas and units to the user\}

* A method named "translate abstraction" is used to inform the user with a translation or explanation of the abstraction in each object class.

\{ User request: Translate Abstraction, check requested abstraction type,

display abstraction declaration to the user\}

Any object such as e_Node inherits all features and capabilities of the "Node" object; in addition to the tree structure allows objects from sub classes to inherit values from super classes. The Node data includes laws and formulas, which are wrapped up in an object for encapsulation purposes.

However, all object classes have the same semantics. Polymorphism allows each of them to carry out operations or methods having the same name in its unique way. An example of this is the "get info" method that acts in different ways according to each instance such as e_Node, $\Omega \_$Node, or $\omega \_N o d e$, since each of them has its own laws and formulas.

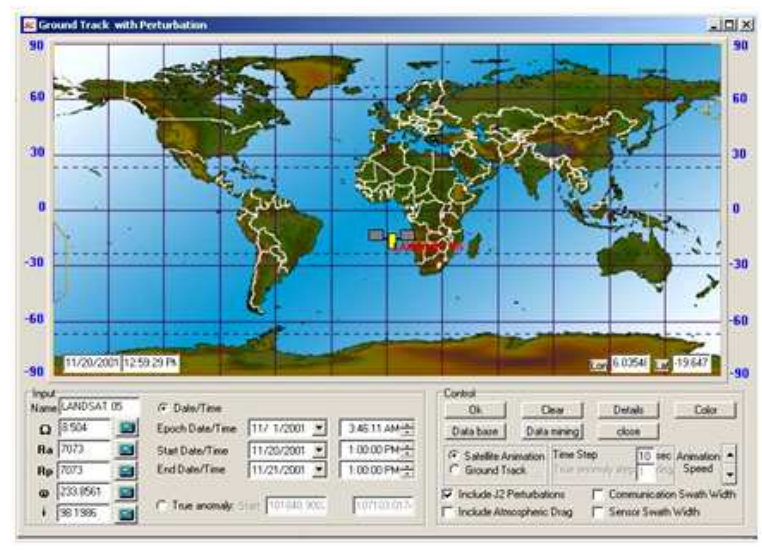

Fig. 3: Ground Track Module (Second Module)

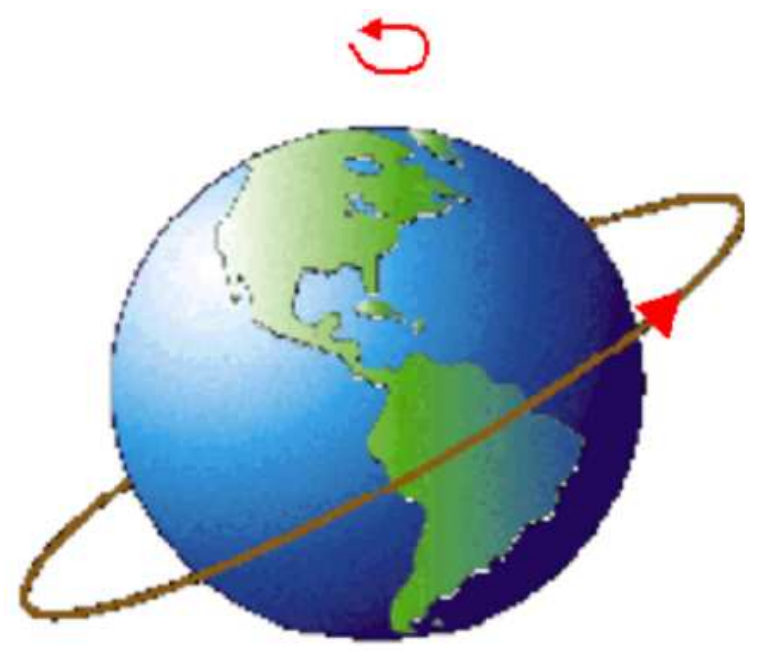

Fig. 4: The Earth and Satellite Motion

Ground Track Module: The Ground Track Module, Fig. 3, is the second module in OOST and it is the most important and complicated module, since it performs the main goals of the system, which is orbit design and simulation. This requires huge computations to be done. The amount of these computations depends on the amount of options required by the user and the period of simulation ${ }^{[12,13]}$.

The Simulation Model: The six orbital elements are enough to visualize orbit shape, orientation and satellite position. The satellite mission designer needs to know well what part of the Earth the satellite is passing over at any given time, at what time the ground stations can access the satellite and other valuable information which can be gained by examining the ground track of the orbit; these can be done by simulating the ground track of the orbit, (Fig. 4).

Ground track is the trace of the path the satellite takes over the Earth's surface. Taking into consideration that the satellite seems as if it is covering the circumference of the Earth each orbit (approximately 
$40000 \mathrm{~km}$ ) and that the Earth is spinning over its axis at speed more than $1600 \mathrm{~km} /$ hour at the equator. Figure 4 shows the Earth and satellite motion.

By Applying this approach, it finds that the ground, Fig. 5, track will stay the same and the satellite will continue to pass all orbits over each other. Even if we change the size and shape of the orbit, the ground track would look the same since it has been considered that satellites rotates around a non-rotating Earth. Figure 5 shows the ground track over a non-rotating Earth.

If the Earth started rotation again, all orbits following the first orbit will be shifted toward the west. This is because the Earth rotates to the east and the orbit plane is fixed in the inertial space, thus the satellite will always return to the same point in the inertial space. Since the Earth rotates at a fixed rate of about 15 degrees/hour $(360 \mathrm{deg}$ in 24hour = $15 \mathrm{deg} /$ hour), this rotation can be taken as a clock used to measure the orbital period. It is required to measure how much the orbit's ground track moves to the west from one orbit to the next. This movement can be quantified as Node Displacement $\Delta \mathrm{N}$, which is measured along the equator from one ascending node to the next ${ }^{[7,8]}$.

Orbital period $(\mathrm{h})=\frac{360-\Delta \mathrm{N}(\text { for direct orbit })}{15 \mathrm{deg} / \text { hour }}$

The ground coordinates of the satellite must be calculated precisely ${ }^{[20,21,22]}$ to simulate the ground track as follows:

Latitude $=\sin ^{-1}[\sin (\mathrm{i}) / \sin ($ ALat $)]$

ALat $=v+\omega$

Where:

i am orbit inclination (deg).

ALat is an Argument of latitude (deg).

$v$ is the true anomaly angle (deg).

$\omega$ is the argument of perigee (deg).

Longitude $=\Omega+\mathrm{Lo}-\mathrm{d} \Omega-\mathrm{Re}$.

Where:

$\Omega$ is the longitude of ascending node (deg).

Lo is the longitudinal angle (deg).

$\mathrm{d} \Omega$ is the regression of nodes since passing the ascending node (deg).

$\mathrm{Re}$ is the Earth rotation since passing the ascending node (deg).

Description of the Ground Track Module: The Ground Track Module is divided into 3 main groups, which are Simulation, Input and Control groups, that work together to visualize the results of calculations and to make the simulation process easy, interactive and realistic.

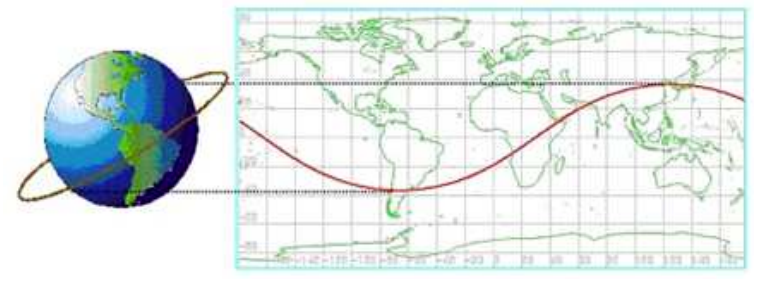

Fig. 5: The Ground Track Over a Non-rotating Earth

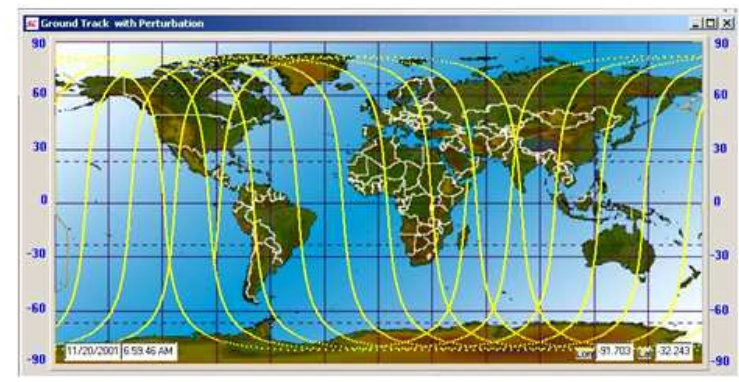

Fig. 6: Ground Track Simulation

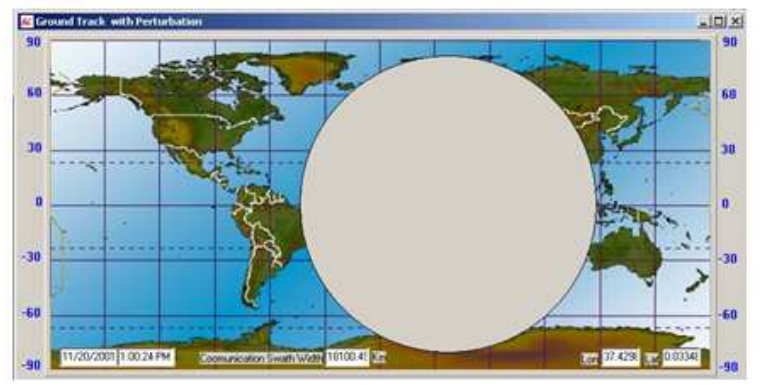

Fig. 7: Communication Swath Width for Nilesat

The Simulation group is composed of an interactive world map showing the value of longitude and latitude for satellites during simulation process, in addition to displaying the changes in simulation date and time.

The simulation group has four displaying modes as follows:

* Satellite animation mode: in this case the simulation shows the satellite animation without drawing the ground track of the satellite on the world map

* Ground Track mode: the simulation shows the ground track of the satellite on the world map (Fig. $6)$.

* Communication swath width mode: the simulation shows a gray circle representing the communication coverage (Fig. 7).

* Imaging sensor swath width mood: the simulation shows a red circle with yellow fill color showing the calculated sensor coverage which depends on the sensor's inclination angle and specifications (Fig. 8). 


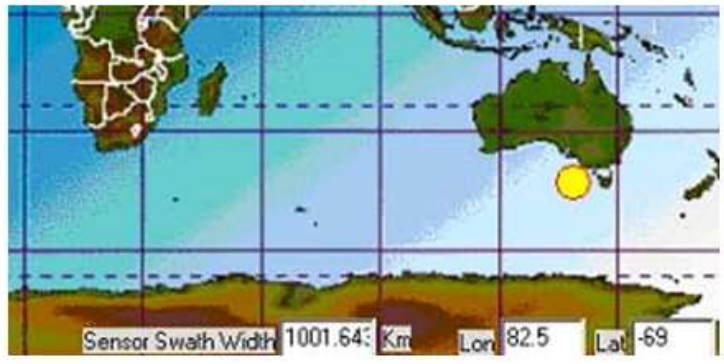

Fig. 8: Imaging Sensor Swath Width

* Both (c) and (d) can be displayed on the same screen and their diameters are displayed in kilometers in the simulation map taking into consideration that circle diameters may vary with time according to satellite altitude.

The input group off the ground track form enables the users to decide whether to start the simulation by changing the True Anomaly value or by specifying both start and end date/time.

The control group of the Ground Track form is responsible for adjusting all settings and options required for simulation. It includes the following operations:

* Selecting between Satellite animation and Ground track.

* Setting the J2 perturbations and Atmospheric Drag on/off.

* Setting the Communication swath width and Sensor swath width on/off.

* Controlling the animation speed and amount of time step or true anomaly step.

* Selecting the ground track color and clearing the simulation group.

* Direct calling for Data base form and data mining form.

\section{RESULTS}

Testing the module results is based on two methods, the first method is through comparing ground track applications results from highly qualified and specialized institutes with results made by using the Ground Track Module. The second method is based on entering orbital elements of well known satellites to both the implemented tool (OOST) and the Satellite Tool Kit (STK), which is one of the most powerful packages in the field of satellite mission design at the current time and then comparing the shape of the resultant orbits in both cases.

Evaluation of Ground track module is based on measuring its capability to solve real problems related to shuttles and well known satellites for different missions such as Science, Remote Sensing, Missile warning, Navigation, ... and others.

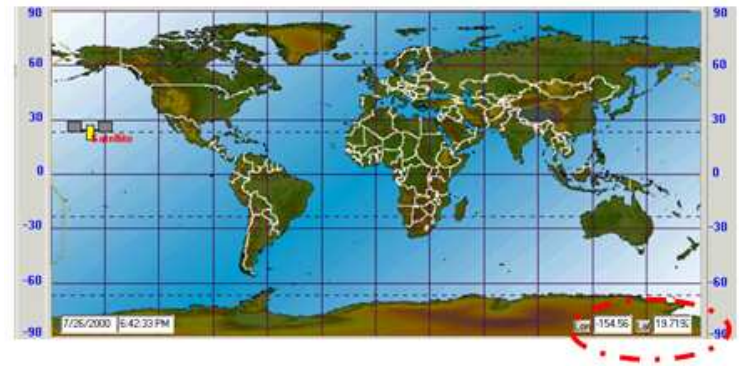

Fig. 9: The Space Shuttle Positioning Application Results Through the Implemented OOST (Red Circle Surrounds the Lon/Lat Values and Satellite Positioning)

The American Institute of Aeronautics and Astronautics (AIAA) has presented the following three applications and their results. Other results have been calculated through the implemented OOST package is presented also for comparison and testing purposes.

The Atlantis Space Shuttle Application: The elements of the May 1989 flight of the Space Shuttle Atlantis (Fig. 9), were as follows:

* $\quad$ Perigee Altitude $=270 \mathrm{~km}$

* $\quad$ Apogee altitude $=279 \mathrm{~km}$

* Eccentricity $=0.000676$ (unit less)

* Semi major axis $=6652,64 \mathrm{~km}$

* Period $=90 \mathrm{~min}$

* Argument of Perigee $=25 \mathrm{deg}$

* Inclination $=28.5 \mathrm{deg}$

* Longitude of ascending node $=167$ deg East

* Find the latitude and longitude of Space Shuttle Atlantis for true anomaly of $20 \mathrm{deg}$.

The final answer presented by the AIAA is as follows:

* $\quad \mathrm{La}=19.72$ deg North , Lo $=154.6$ deg west

The Landsat Satellite Swath Width Application: The Landsat D is in a circular, near polar Earth orbit at an altitude of $709 \mathrm{~km}$. What is its communication swath width?

The final answer presented by the AIAA is: $\mathrm{SW}=$ 5754 km (Fig. 10).

The Landsat D Satellite Field of View Application: The Landsat D (circular orbit $.709 \mathrm{~km}$ altitude ) multi spectral scanner has a field of view of $14.90 \mathrm{deg}$ centered on the nadir what is the swath width of the instrument?

The final answer presented by the AIAA is: $\mathrm{S}_{\mathrm{i}}=$ 185.7 km (Fig. 11).

The IUE American Satellite for Scientific Experiments and the Russian Missile Warning Satellite Cosmos 2340 Application : By comparing 
the orbits shapes of both IUE scientific experiments satellite and Cosmos 2340 missile warning satellite implemented by both STK and OOST software packages, it has been found that both of them give the same orbit shape. Figure 12-15 show IUE and Cosmos 2340 Orbits shapes implemented by both packages.

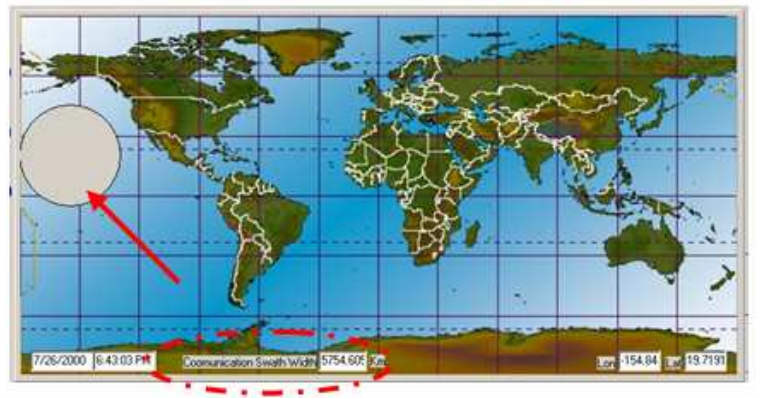

Fig. 10: Landsat Communication Swath Width Application Result Through the Implemented OOST (Red Circle Surrounds the Communication Swath Width Value)

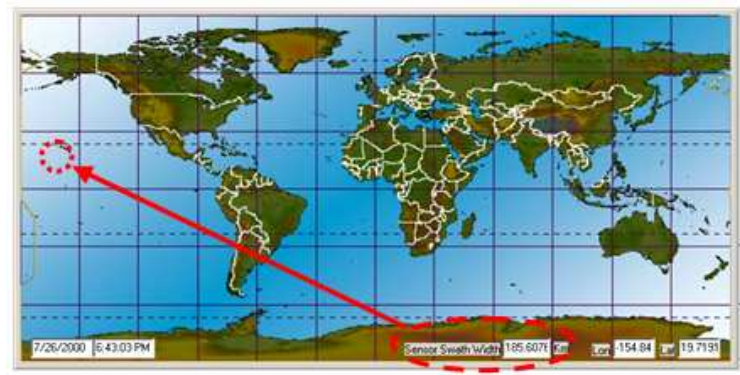

Fig. 11: Landsat Field of View Application Result Through the Implemented OOST (Red Circle Surrounds Sensor Field of View Value and Satellite Position)

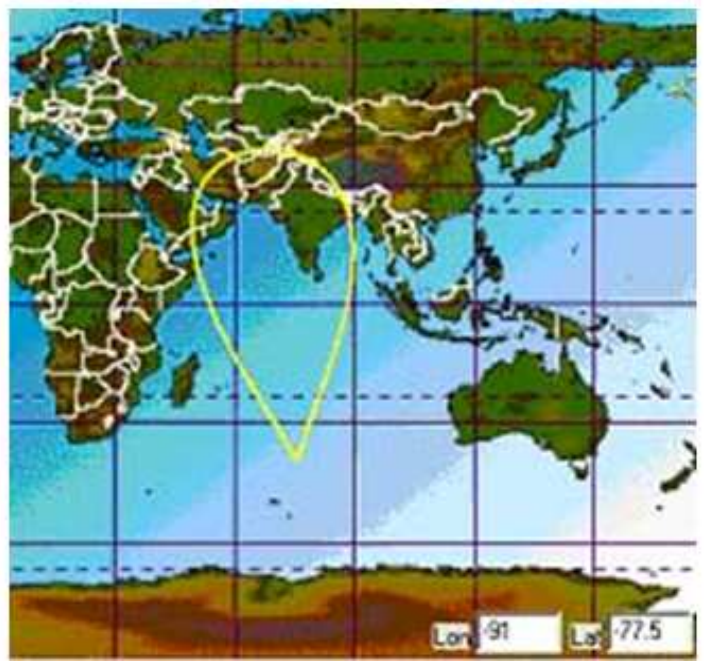

Fig. 12: UE Satellite Orbit Shape with the Implemented OOST
The Orbit Analysis Module: This is the third module of OOST and it aims at:

* Building orbital elements database for real satellites.

Developing the established database with some intelligent features by making use of a suitable Data Mining technique ${ }^{[14-16]}$.

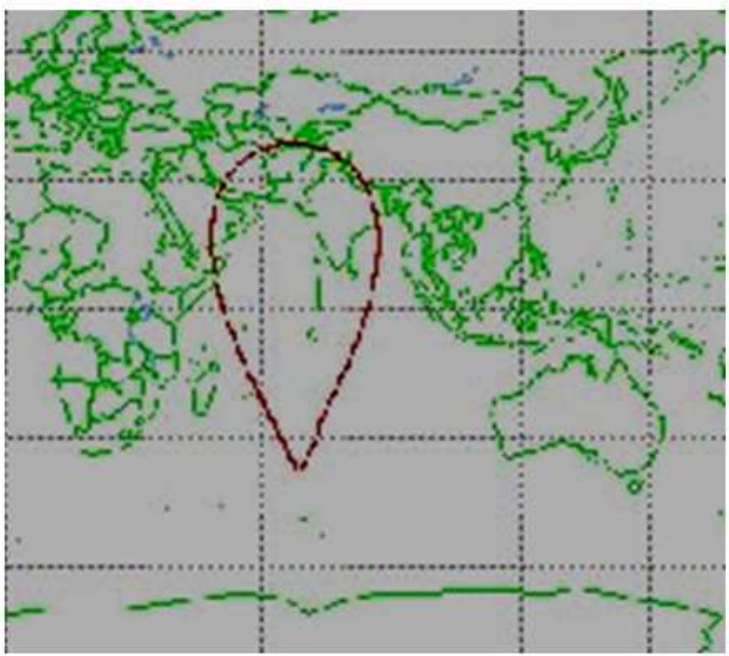

Fig. 13: IUE Satellite Orbit Shape with STK Package

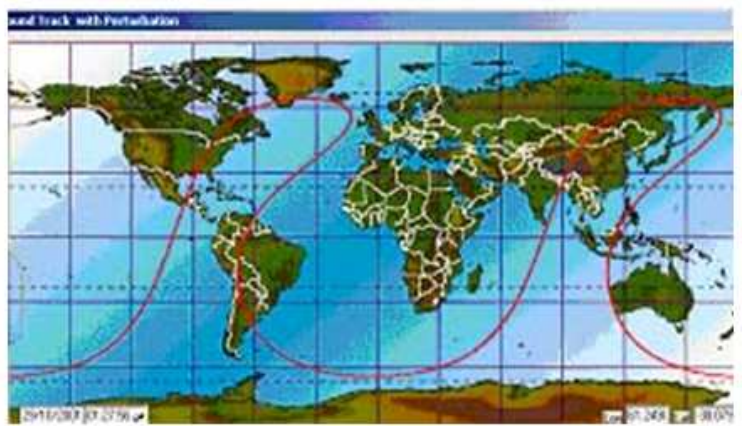

Fig. 14: Cosmos 2340 Satellite Orbit Shape with the Implemented OOST

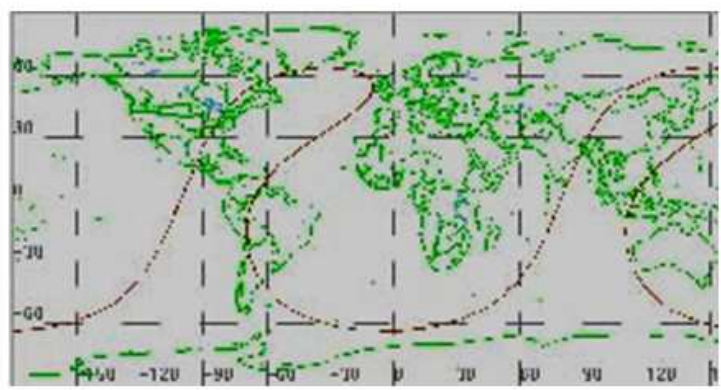

Fig. 15: Cosmos 2340 Satellite Orbit Shape with STK Package 
* Classifying different orbit designs of various missions into known classes and special case classes, then doing a complete study for the special case classes in order to predict abnormal orbits and applications. Accordingly one can detect and analyze different trends for satellites owners.

Data Mining: Military and Remote Sensing satellite missions has been taken for study by using the Decision Tree technique, in order to gain some valuable information about these missions. This information can be made by Classifying different orbit designs into known classes and special case classes, then doing a complete study for special case classes, in order to predict abnormal orbits and applications and to detect and analyze different trends for satellites owners.

Patterns covering satellite missions in the area of interest will be generated. These patterns are hidden deep in the satellite database and it is not obviously mentioned in any of its fields. The OOST will make an efficient probing for satellite data by generating a broad range of summaries and recognizing relationships among different orbital element records.

Two types of Knowledge have been used. The first is user-defined vocabulary that provides relational views of data and is used to express generalization relationships among different data types. For example, the mission designer can define "Mill" as an orbit for a satellite whose inclination is between 63 and 105 degrees and its mission is military. The second type is how to use abstracts that are summaries of the data expressed in terms of this vocabulary. The vocabulary and abstracts deliver the system with the ability to search for patterns in terms of sets that are meaningful to the user. For example the "Geosynchronous" attribute which is an orbit whose nearer altitude (Perigee) and fairest altitude (Apogee) are between 35000 and $36000 \mathrm{~km}$, can be a set of [Geosynchronous communication, Missile warning] missions.

A correctness measure is applied to measure the strength or the level of certainty each pattern holds. In addition of a data dictionary that contains vocabulary of the mission designer predicates, a classification hierarchy for each separate mission and a set of abstraction functions have been also established.

The Military Satellites Data Mining: The classification hierarchy for Military Satellites Mission (Fig. 16) includes four levels. The Vocabulary "Military Sat" represents the main node of the Military decision tree. This node is split in the second tree level into 3 classes represented by 3 vocabularies [Mil1, Mil2 and Mil3]. In the third tree level, Mil1 is split into 3 classes [Mil1 O-1, Mil1 O-2, Mil1 O-3,]. Each of Mil2 and Mil3 has only one class represented by 2 vocabularies [Mil2 $\mathrm{O}-1$ and Mil3 $\mathrm{O}-1$ ] respectively. In the fourth tree level Each of Mil2 O-1 and Mil3 O-1 is split into 4 levels [LEO, MEO, GEO, Molnya]. A detailed explanation of these classes discussed.
Since orbit inclination is an important attribute in case of military missions, due to its effect on imaging coverage, it has been chosen to be the main splitter in the military satellites decision tree. The "Military Sat" Node selects satellite records whose Mission= military grouped by orbit inclination. This grouping results 30 military satellite record classified into 3 classes Mil1, Mil2 and Mil3.

The Mil1 node represents the "Normal Earth Coverage" trend ${ }^{[20,22]}$ covered by the majority of military satellites (25 satellite $-83 \%)$. Grouping the Mil1 node by the "owners" attribute in the next tree level will lead to detecting the satellite owners following this trend. Thus the Node Mil1 is classified into 3 leaves (Mil1 O-1, Mil1 O_2, Mil1 O-3) representing the 3 military satellite owners following this trend (USA, USSR, CIS).

The Mil2 node represents the "Special Earth Coverage" trend for satellites orbiting in the direction of earth rotation (Prograde orbits). This trend is covered by 4 satellites representing $13 \%$ of military satellites. The Mil2 node is grouped by "owners" to detect owners of this trend, It has been found that all the 4 satellites are owned by USA (node Mil2 O-1). In the next tree level, three attributes have been chosen to act as main splitter. These attributes are both 'Apogee' and 'Perigee' altitudes in order to detect the nearest and farthest altitudes of the working orbit; in addition of the ratio between Apogee and Perigee in order to detect whether it is Molnya orbit or not. By reaching this three level, OOST can discover the suitable missions for these orbits; in addition to analyzing its advantages and disadvantages and evaluating the whether the 'mission' attribute delivered from the database is true or false. It has been discovered that these 4 satellites have abnormal orbits.

Study and analysis of this trend are based on grouping 'inclination' attribute to reach primary estimation for each satellite mission, then grouping 'owners' attribute to know owners following this trend, finally grouping 'Apogee' and 'Perigee' attributes to discover and confirm the true satellite mission in addition of classifying orbits into classes and assigning each orbit to its suitable class or category.

The Mil3 node also represents a "Special Earth Coverage" trend like Mil2, but for satellites orbiting opposite to the earth rotation direction (Retrograde orbits). In the next tree level (node Mil3 O-1), it has been found that this trend is covered by only one satellite representing $3 \%$ of military satellites and it is owned by Israel. In the next tree level, the same three attributes used with Mil2 $\mathrm{O}-1$ have been chosen to act as a main splitter with Mil3 O-1; and for the same purposes. By reaching this three level, OOST can analyze the true mission of each satellite. Study and analysis of this trend are based on the same procedures like Mil2. 


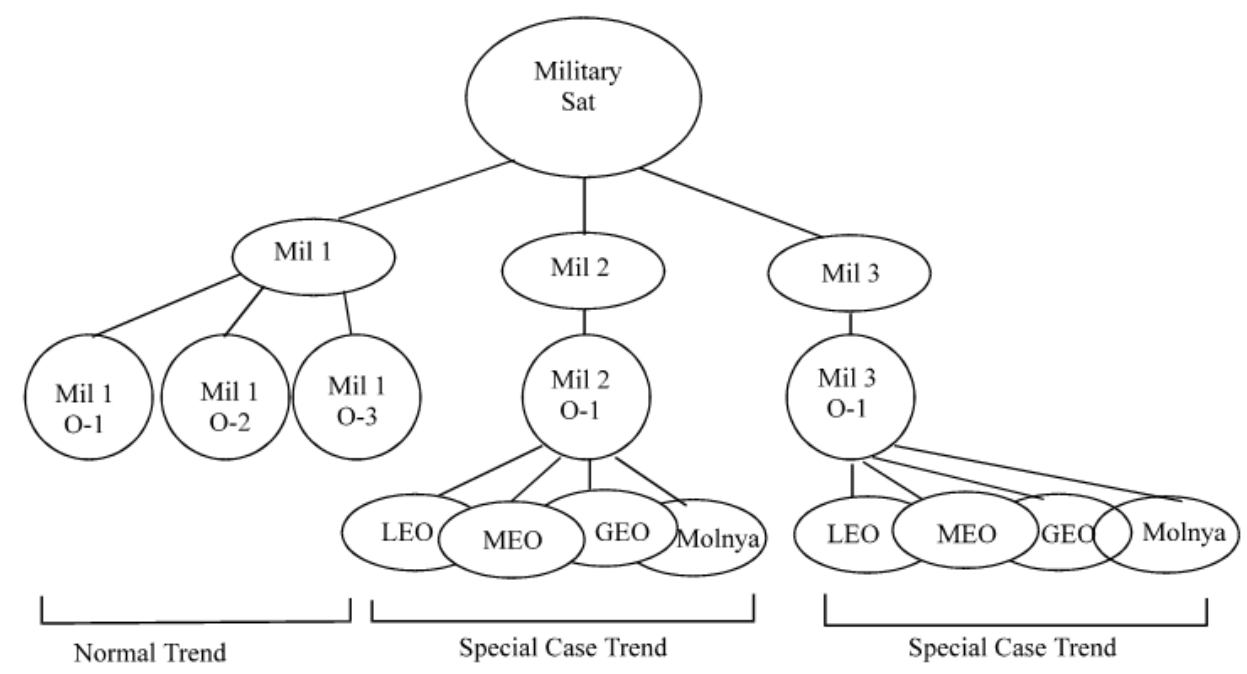

Fig. 16: Classification Hierarchy for Military Satellites Mission

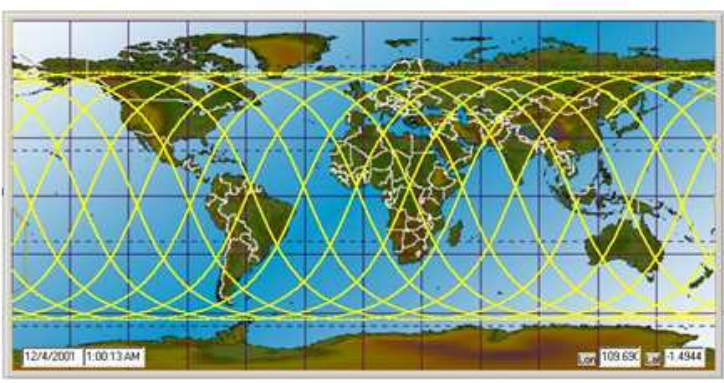

Fig. 17: OOST Implementation of the Normal Earth Coverage Trend of the Military Satellite USA 122 of the Mil1 Node

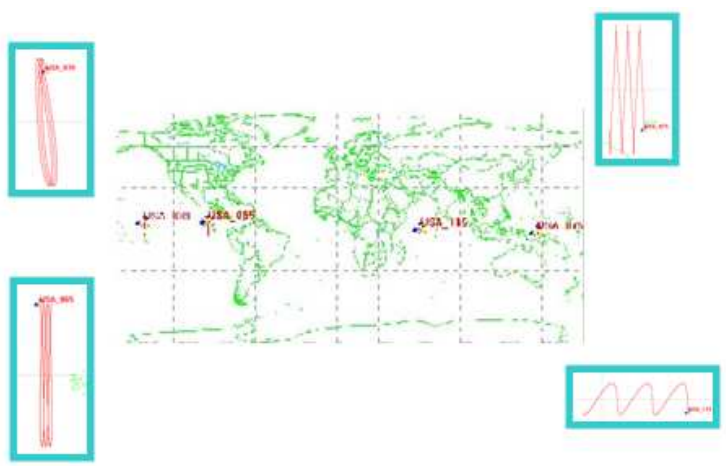

Fig. 18: Special Earth Coverage Satellites of the Mil2 Node

\section{Mil1 Node : (Normal Earth Coverage Trend)}

Vocabulary: Mill: (63 deg <= orbit inclination $<=105$ deg) and Mission=Military

Pattern: The Mill satellites are always Military satellites with normal earth coverage:

Mil1 $\rightarrow$ Military satellites with normal earth coverage (with strength $100 \%$ )
Trend Analysis: Mill Node represents the normal coverage requirements for most of the military satellites (25 satellites from 30 military satellites) (Fig. 17.) It satisfies many advantages that represent a common requirement for the majority of users. However military satellites work in Low Earth Orbits (LEO) and seem to be normal imaging satellites like Remote Sensing satellites, but in fact, this trend shows many different characteristics as follows:

* It does not need in most cases to be sun synchronous in order not to cover areas of interest at fixed time daily. But it may need to do its mission in many different times to help in monitoring targets.

* It may need in most cases to increase the revisit time and to concentrate on certain areas of interest, not to have a global coverage like commercial remote sensing satellites that have a near polar inclination, this can be done by decreasing the inclination with a limit that allow them to cover their targets (example: allowing both USA and Russia to cover each other).

Mil2 Node:

Vocabulary: Mil2: orbit inclination $<63 \mathrm{deg}$ and Mission=Military

Pattern: The Mil2 satellites are mostly either Military Missile Warning satellites or Military Communication satellites and their true mission is not really mentioned in the satellite database (They are not military imaging satellites) (Fig. 18).

Mil2 $\rightarrow$ Military Missile Warning/Communication satellites (with strength 100\%)

Trend Analysis: The Mil2 node represents the "Special Earth Coverage" trend for satellites orbiting in the 
direction of earth rotation (Prograde orbits). By applying the previously discussed sequence of grouping and classifications, a detailed analysis of the Mil2 satellite missions have been established, it has been discovered that the 4 satellites represented by this node have an abnormal orbits that makes them not capable of capturing images for military purposes; this is due to their high altitude, low inclination angle and large orbital period since they work on equatorial geostationary orbits. Thus they are mostly estimated to be either missile warning satellites or Military Communication satellites with hidden mission information.

By allocating different issued information about these satellites, it has been found that three of the 4 satellites (USA 39,65,115) belong to the Defense Support Program (DSP) for missile warning purposes (Federal Association of scientist, www.fas.org) and the fourth is Secure Military communication satellite (Encyclopedia Astronautica, www.astronautix.com). Calculating the level of certainty of this American trend leads to reaching $100 \%$ strength. Figure 18 shows magnified images for the 4 satellite orbits.

\section{Mil3 Node:}

Vocabulary: Mil3: orbit inclination > $105 \mathrm{deg}$ and Mission=Military

Pattern: The Mil3 satellites are always Military retrograde satellites with special earth coverage:

Mil3 $\rightarrow$ Military retrograde satellites with special earth coverage (with strength $100 \%$ )

Trend Analysis: By applying the sequence of grouping and classifications described in the classification hierarchy, a detailed analysis report has been generated by OOST for the Mil3 Ofeq-5 Israeli satellite mission. As shown in Fig. 19, it includes the following:

* Its areas of interest are the Middle East and/or equatorial areas, since its inclination is $144 \mathrm{deg}$, allowing nadir coverage for areas between +36 or 36 deg Latitude only, instead of being in a near polar orbit with global coverage.

* High revisit time, since it has lowest orbit inclination compared with all other military satellites working in low earth orbit.

* Retrograde orbit is faster orbiting velocity, since it rotates opposite to the direction of earth rotation; this helps in increasing revisit time and making its earth detection more difficult.
* It can produce high resolution images since it is very close to earth. Its orbit is elliptical with perigee equal to $358 \mathrm{~km}$.

* It can capture images in many different times to help in monitoring targets instead of being limited with fixed time as in the case of sun synchronous orbits.

Abstraction: An abstraction representing the latitude coverage, resulted from the Mil1, Mil2 and Mil3 class types is shown in Table 1.

Remote Sensing Satellites Data Mining: The classification hierarchy for Remote Sensing Mission ${ }^{[19-}$ 22] includes four levels, (Fig. 20). The Vocabulary "Remote Sensing" represents the main node of the Remote Sensing decision tree. This node is split in the second tree level into 2 classes represented by 2 vocabularies [RS1, RS2]. On the third level, RS1 is split into 13 leaves [RS1 O-1 to RS1 O-13] and RS2 is split into 3 nodes [RS2 O-1 to RS2 O-3] each of them is split in the fourth tree level into 4 levels [LEO, MEO, GEO, Molnya]. The detailed explanation of these classes is discussed.

As in Military satellites mission, Orbit inclination attribute has been chosen again to act as the main splitter in the remote sensing satellites decision tree, since both military and remote sensing missions do the same function, which is capturing images, but with completely different specifications and objectives; in addition to the limitation of the vertical latitudes coverage, which is restricted by the inclination angle (For example, if the inclination is 35 degree, this will restrict the vertical (nadir) coverage to be limited in an area between (+ or -) 35 degree latitude). The root Node named "Remote Sensing" selects satellite records whose Mission = Remote Sensing, grouped by orbit inclination. This grouping results 38 Remote Sensing satellite records.

The RS1 node represents the trend of "Normal Sun Synchronous Orbits"; this trend is followed by the majority of commercial remote sensing satellites (33 satellites representing $85.84 \%$ of remote sensing satellites).

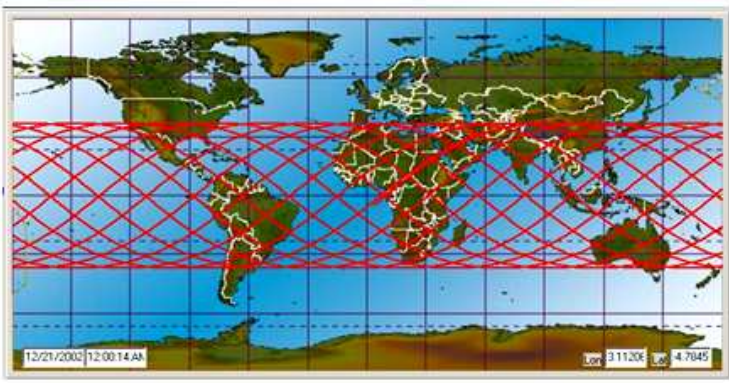

Fig. 19: OOST Implementation of the Special Retrograde Coverage Trend for the Military Satellite Ofeq5 of the Mil 3 Nodes 
Table 1: Abstraction of Military Latitude Coverage

\begin{tabular}{|c|c|c|c|c|c|c|c|c|c|}
\hline \multicolumn{10}{|c|}{ Military latitudes coverage (deg) } \\
\hline Class type & Mission & Coverage & Latitudes & $\begin{array}{l}\text { Direction } \\
\text { of followers }\end{array}$ & Number & LEO & MEO & GEO & Molnya \\
\hline Mil1 Military & Normal & 63 to 105 & Both & 25 & 25 & 0 & 0 & 0 & \\
\hline Mil2 Military & Special & $<63$ & Prograde & 4 & 0 & 0 & 4 & 0 & \\
\hline Mil3 Military & Special & $>105$ & Retrograde & 1 & 1 & 0 & 0 & 0 & \\
\hline
\end{tabular}

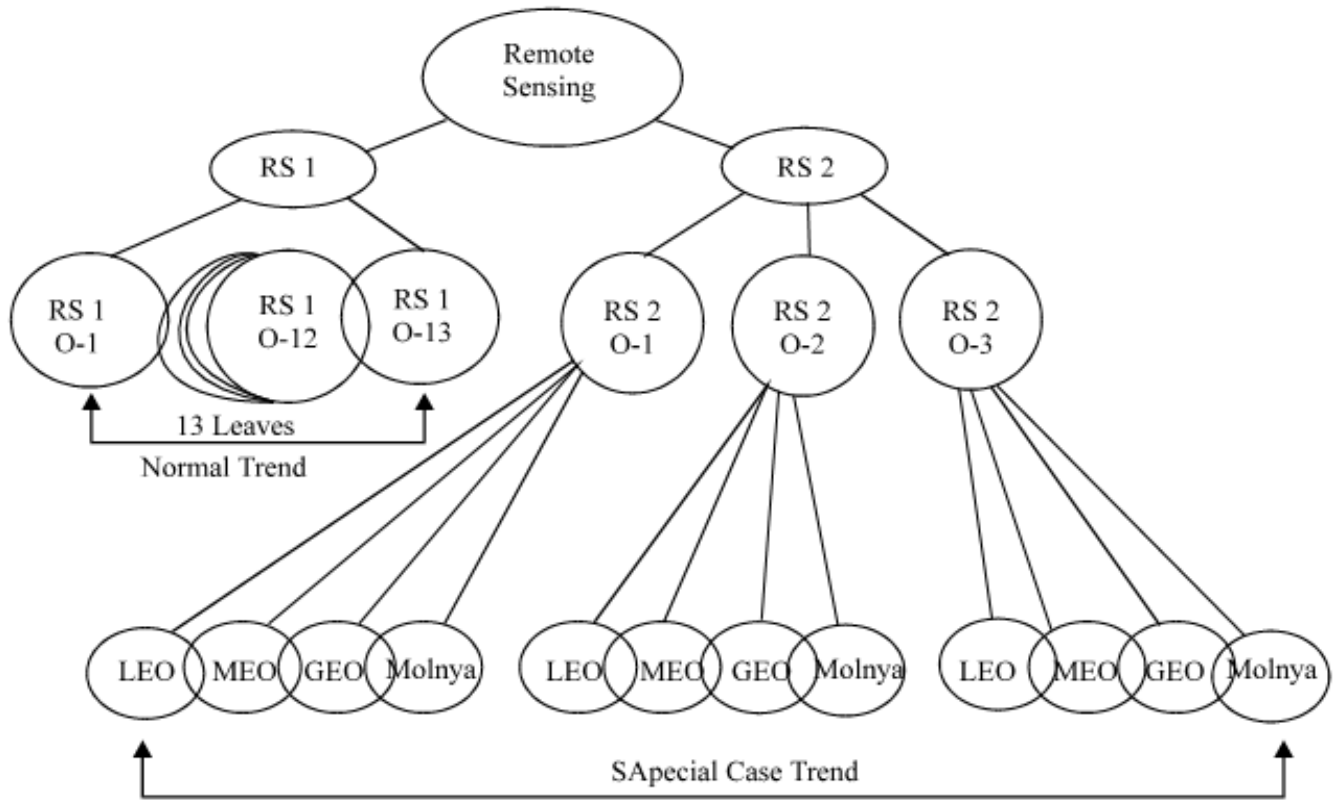

Fig. 20: Classification Hierarchy for Remote Sensing Mission

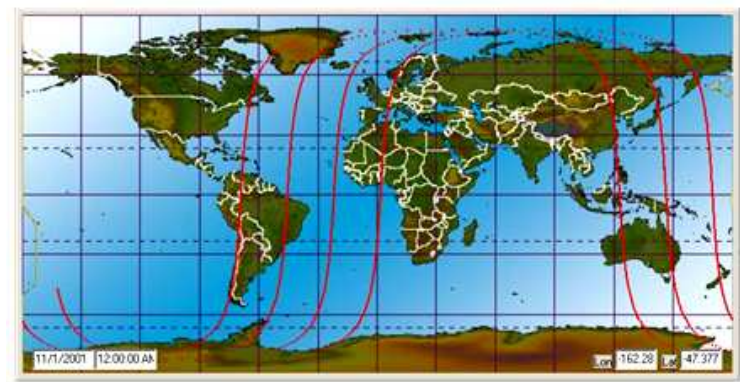

Fig. 21: OOST Implementation of the Normal Sun Synchronous Orbit With Global Coverage Trend for Quick Bird 2 Satellite

Grouping the RS1 node by the "owners" attribute in the next tree level will lead to detecting the satellite owners following this trend. Thus the Node RS1 is classified into 13 leaves (RS1 O-1 to RS1 O-13) representing the 13 owners following this trend.

The RS2 node represents the "Special non Sun Synchronous Orbits" trend; this trend is followed by the minority of remote sensing satellites (only 5 satellites representing $13.16 \%$ of remote sensing satellites). The RS2 node is grouped by the "owners" attribute to detect the follower of this trend; it has been found that the 5 satellites are owned by Saudi Arabia, USA and CIS (node RS2 O-1 to RS2 O-3). In the next tree level, the same attributes used as a main splitter in the military satellite decision tree are used again since both of them are imaging satellites. Thus leading to know the altitude of the working orbit (LEO, MEO, GEO, Molnya). By reaching this three level, OOST can discover the suitable missions for these orbits; in addition to analyzing its advantages and disadvantages and evaluating the whether the 'mission' attribute delivered from the database is true or false. It has been discovered that these 5 satellites have special applications that are greatly different from known commercial remote sensing satellites.

Study and analysis of this trend are based on grouping 'inclination' attribute to reach primary estimation for each satellite mission, then grouping 'owners' attribute to know owners following this trend, finally grouping 'Apogee' and 'Perigee' attributes to discover and confirm the true satellite mission. This is done through classifying orbits into classes and assigning each orbit to its suitable class or category. A detailed report is generated automatically by OOST for each satellite following this trend.

\section{RS1 Node:}

Vocabulary: RS1: (97 deg <= orbit inclination $<=101$ deg) and mission $=$ Remote sensing. 
Table 2: Abstraction of Remote Sensing Latitudes Coverage

\begin{tabular}{|c|c|c|c|c|c|c|c|c|}
\hline \multicolumn{9}{|c|}{ Remote sensing latitude coverage (deg) } \\
\hline $\begin{array}{l}\text { Class Mission } \\
\text { Type }\end{array}$ & Coverage & $\begin{array}{l}\text { Latitudes } \\
\text { orbit }\end{array}$ & $\begin{array}{l}\text { Sun-synchronous } \\
\text { followers } \\
\text { (total 38) }\end{array}$ & Number of & LEO & MEO & GEO & Molnya \\
\hline $\begin{array}{ll}\text { RS1 Remote } \\
\text { sensing }\end{array}$ & Normal & 97 to 101 & Yes & 33 & 33 & 0 & 0 & 0 \\
\hline $\begin{array}{r}\text { RS2 Remote } \\
\text { sensing }\end{array}$ & Special & $<97$ or $>101$ & No & 5 & 3 & 0 & 2 & 0 \\
\hline
\end{tabular}

Pattern: The RS1 satellites are always Remote Sensing satellites in normal sunsynchronous orbit and Global coverage.

RS1 $\rightarrow \quad$ Remote Sensing satellitesinh normal sunsynchronous orbit and Global coverage (with strength $100 \%$ ).

Trend analysis: The Node RS1 represents the requirements of the majority of Remote Sensing satellites (33 satellites from 38 remote sensing satellites), which normally work in sun synchronous Low Earth Orbit (LEO). Sun-synchronous orbit is an earth orbit with the property by providing a constant sun angle for the observation of the earth, Fig. 21. A constant sun angle is very desirable for cameras and other instruments that observe reflected light. Most of the remote sensing satellites prefer to capture images with sun angle equals to $45 \mathrm{deg}$ in order to get a suitable range of shadow that enhances the quality of the image and makes interpretation easier. A low quality image will result in case of choosing the low sun angle, since the shadow will be too long and also in case of high sun angle such as $90 \mathrm{deg}$, since the resulted image will have no shadow. In sunsynchronous orbit the satellite passes over any particular ground site at the same solar time each day (one pass in the day and one at night).

Most of the remote sensing satellites are commercial satellites; this fact leads to the need of covering the whole earth or most of it, thus mission designers usually increase the inclination angle (around 90 deg.) Making the orbit near polar, in order to allow the satellite to reach higher altitudes.

\section{RS2 Node:}

Vocabulary: RS2: (inclination < 97 or inclination > 101) and mission $=$ Remote sensing.

Pattern: The RS2 Satellites are usually Remote Sensing satellites with non-sun synchronous orbits and special coverage.

RS2 $\rightarrow \quad$ Remote Sensing satellites with nonsun synchronous orbits and special coverage. They are used for Atmospheric, Climate studies and special scientific experiments or other special purposes. (With strength $60 \%$ ).

Trend analysis: By applying the sequence of grouping and classifications described in the classification hierarchy, a detailed analysis reports have been generated by OOST from the 5 satellites following this trend. The first two satellites were analyzed by OOST as Russian Missile Warning satellites. The last three satellites were analyzed in the OOST report as either Atmospheric and Climate studies or special scientific experiments.

Cosmos_2224 and Cosmos_2209 Russian Satellites: In geostationary orbits, if the orbit plane is not in the equatorial plane, a north-south figure eight motion will be produced. Correcting this error requires a simple plane change maneuver. This explains what has happened to the Cosmos_2224 and 2209 satellites. In addition to geostationary orbit is not suitable for remote sensing, this orbit must be used for military communication or missile warning and not for remote sensing as it is mentioned in the database. So the real mission is hidden and replaced by Remote Sensing mission. The OOST analysis matched with information issued in www.clw.org (Coalition of Reducing Nuclear Danger).

The other three special orbits belonging to the USA and Saudi Arabia are in fact remote sensing orbits but they are not sun synchronous and they have low inclinations, which depend on their different special application as follows:

TRMM American Satellite: It represents tropical rainfall monitoring mission for tropical areas with inclination $35 \mathrm{deg}$. Thus it needs to cover tropical areas only instead of having a global coverage. The OOST analysis matched with information issued in www.eoc.nasda.gov.jp (Japanese Space Agency).

Orbview-01 American Satellite: It is used to study spatial and temporal distribution of global lightening for predicting major atmospheric storms and climate changes. Its inclination is $70 \mathrm{deg}$ to work in area between $-70 \mathrm{deg}$ and $+70 \mathrm{deg}$ latitude. The OOST analysis matched with information issued in www.orbimage.com (Orbital Imaging Corporation)

Saudisat -1A Saudian Satellite: It represents a special scientific applications satellite used for performing different scientific experiments. The OOST analysis matched with information issued in www.kacst.edu.sa (King Abdul Aziz city for Science and Technology). 
Abstraction: an abstraction representing the latitude coverage, resulted from the RS1 and RS2 class types is shown in Table 2.

\section{DISCUSSION}

Two types of missions have been taken for study using Decision Tree Technique (Military, Remote Sensing). Two types of Knowledge have been used. The first is user-defined vocabulary that provides relational views of data and is used to express generalization relationships among different data types. The second type is how to use abstracts that are summaries of the data expressed in terms of this vocabulary. The vocabulary and abstracts deliver the system with the ability to search for patterns in terms of sets that are meaningful to the user.

Military satellites are classified into three classes as follows:

* Class 1: Represents the "Normal Earth Coverage" trend covered by the majority of military satellites (25 satellite $-83 \%$ ). Satellite owners following this trend are (USA, USSR, CIS).

* Class 2: Represents the "Special Earth Coverage" trend for satellites orbiting in the direction of earth rotation (Prograde orbits). This trend is covered by 4 satellites representing $13 \%$ of military satellites. It has been found that all the 4 satellites are owned by USA.

* Class 3: Represents the "Special Earth Coverage" trend for satellites orbiting opposite to the earth rotation direction (Retrograde orbits). This trend is covered by only one satellite representing $3 \%$ of military satellites. It has been found that Israel is the only follower of this trend and that it has a unique orbit that supplies its follower with many valuable advantages that satisfies the owner's unique and special requirements.

Remote Sensing satellites are classified into two classes as follows:

* Class 1: Represents the trend of "Normal Sun Synchronous Orbits". This trend is followed by the majority of commercial remote sensing satellites (33 satellites representing $85.84 \%$ of remote sensing satellites). It has been found that 13 remote sensing satellite owners following this trend

* Class 2: Represents the "Special non Sun Synchronous Orbits" trend, followed by the minority of commercial remote sensing satellites (only 5 satellites representing $13.16 \%$ of remote sensing satellites). It has been found that the 5 satellites are owned by Saudi Arabia, USA and CIS. They have special applications that are greatly different from known commercial remote sensing satellites.

\section{CONCLUSION}

This study presents OOST as an object oriented tool for satellite orbit design with various significant capabilities. Such capabilities are pointed out in the following:

* It includes an orbital computing environment that acts as a simple interactive facility for orbital calculations. The User Guiding Trees, UGTs, act as an interactive tool that can help choosing the most convenient method for orbital calculation.

* Ground tracking satisfies the following goals for all types of geocentric orbits:

* Satellites animation simulation.

* Ground track simulation.

* Communication swath width simulation.

* Sensor field of view simulation.

* J2 perturbation calculation.

* Atmospheric Drag calculation.

* All simulations based on changing either the real date/time or true anomaly.

* Direct connection between Ground Track Module and both satellite database and Data mining forms allows users to perform tests on real satellite data.

* Decision Tree technique succeeds in discovering valuable data related to classifying satellites and defining the trends of their owners. This classification is based on classifying satellite orbits into two trends, one representing the majority of owners and the other represents special cases. A report is generated for special cases orbits by OOST. These reports can be summarized as follows:

* The USA 39, 65, 75 and 115 satellites owned by USA and Russian satellites Cosmos 2209 and 2224 are not capable of capturing images, this is due to their high altitude, low inclination and large orbital period as they work on equatorial geosynchronous orbits. Thus they are either missile warning or military communication satellites with hidden mission information.

* The Ofeq 5satellite owned by Israel has a unique orbit which supplies many valuable advantages that satisfy the owner's unique and special requirements as follows:

* Area of interest is between +36 or -36 degree latitude due to the low orbit inclination which leads to high revisit time.

* Retrograde orbit has a faster orbiting velocity which leads to high revisit time and difficult ground detection.

* The perigee height is very low which leads to high resolution images since it is very close to earth.

* Capturing images in non fixed time instead of fixing the time as in the case of sun 
synchronous orbits, leads to support monitoring targets.

* The TRMM, Saudisat 1A and Orbview 01 satellites have special remote sensing missions such as science experiments, tropical rainfall monitoring, distribution of lightening.

\section{REFERENCES}

1. Charles, D. B., 1998. Spacecraft Mission Design. Second Edn. American Institute of Aeronautics and Astronautics.

2. James, R. Wertz and Wiley J. Larson, 1999. Space Mission Analysis and Design. Space Technology Library.

3. Dan, L. Boulet, 1991. Methods of Orbit Determination for the Microcomputer. WillmannBell Inc.

4. Pedro Ramon Escobal, 1976. Methods for Orbit Determination. Krieger Publishing, Florida.

5. Jerry Jon Sellers, 1994. Understanding Space. McGraw-Hill Inc.

6. Richard G. Madonna, 1997. Orbital Mechanics. Krieger Publishing, Florida.

7. Tom Logsdon, 1998. Orbital Mechanics. John Wiley and Sons Inc.

8. Chobotov, V.A., 1991. Orbital Mechanics. American Institute of Aeronautics and Astronautics.

9. Tom Swan, 1998. Delphi 4 Bible. IDG Books Worldwide.

10. Bruno, R. Preiss, 1998. Data Structure and Algorithms Using Object Oriented Design. Addison Wesley Publishing, Canada.

11. Said, M. Abdel-Wahab and Ahmed Mohamed Hamad, 2003. Interactive Visualization of Satellite Footprint in Simulated Environment. $10^{\text {th }}$ International ASAT Conference, Cairo.
12. David Baker, 2002. Janes Space Directory 2002/2003. Jane's Information Group Limited.

13. Mark Long, 1988. World Satellite Almanac. $21^{\text {st }}$ Century Publishing.

14. Michael, J.A. Berry and Gordon Linoff, 1997. Data Mining Techniques For Marketing, Sales and Customer Support. Wiley Computer Publishing.

15. Vasant Dhar and Alexander Tuzhilin, 1993. Abstract-driven pattern discovery in databases. IEEE Trans. On Knowledge and Data Eng., 5: 6.

16. Rakesh Agrawal, Tomasz Imielinski and Arun Swami, 1993. Database mining: A performance perspective. IEEE Trans. On Knowledge and Data Eng., 5: 6.

17. Fabienne Delhaise, 1991. Analytical Treatment of Air Drag and Earth Oblateness Effects Upon an Artificial Satellite. Facultes N.D de la Paix, Belgium.

18. Mavraganis, A.G. and D.G. Michalakis, 1992. The Two-body problem with drag and radiation pressure. National Technical University of Athen.

19. Joel Alper and Joseph N. Pelton, 1984. The Intelsat global satellite system. American Institute of Aeronautics and Astronautics.

20. Paul, J. Curran, 1985. Principles of remote sensing. Longman Scientific and Technical.

21. Michael Hord, R., 1986. Remote Sensing Methods and Applications. John Wiley and Sons Inc.

22. Anonymous, 1998. American Ephemeris and Nautical Almanac, U.S. Government Printing Office, Washington. 